\title{
Minimum emittance in storage rings with uniform or nonuniform dipoles
}

\author{
Chun-xi Wang* \\ Argonne National Laboratory, 9700 South Cass Avenue, Illinois 60439, USA
}

(Received 18 March 2009; published 3 June 2009)

\begin{abstract}
A simple treatment of minimum emittance theory in storage rings is presented, favoring vector and matrix forms for a more concise picture. Both conventional uniform dipoles and nonuniform dipoles with bending radius variation are treated. Simple formulas are given for computing the minimum emittance, optimal lattice parameters, as well as effects of nonoptimal parameters. For nonuniform dipoles, analytical results are obtained for a three-piece sandwich dipole model. Minimization of the effective emittance for light sources is given in detail. Usefulness of gradient and/or nonuniform dipoles for reducing the effective emittance is addressed.
\end{abstract}

\section{INTRODUCTION}

Low-emittance storage rings play a critical role in electron machines from modern storage rings in synchrotron radiation light sources to the damping rings in the envisioned International Linear Collider. Low-emittance bending cells are also important for preserving beam quality in the arcs of energy-recovery linac-based light sources. Well over a quarter century ago, it was realized that there is a theoretical minimum emittance achievable in electron storage rings with uniform bending magnets via the balance between radiation damping and quantum excitation [1]. Thorough analyses have been done to elucidate this fundamental property of electron storage rings [2-4]. Furthermore, emittance optimization for isochronous rings and optimization of the effective emittance and beam size in straight insertions have been studied [5-7] for light-source developments. More recently, in order to break the theoretical minimum emittance barrier for lowering emittance, nonuniform dipoles with bending radius variation have been considered for emittance minimization [8-11]. Here, we present an alternative treatment for emittance minimization in uniform and nonuniform dipoles, favoring vector and matrix forms for a more concise picture. Simple formulas are given for computing the minimum emittance and associated optimal lattice parameters, as well as effects of nonoptimal lattices. New analytical results on emittance minimization in storage rings with nonuniform dipoles are given. A simple derivation of the minimum effective emittance is presented. Effects of the damping partition and nonuniform dipole on the effective emittance are investigated.

Assuming negligible insertion-device contributions to the radiation integrals, the horizontal natural emittance and beam energy spread in electron storage rings are given by [12]

\footnotetext{
*wangcx@aps.anl.gov; http://www.aps.anl.gov/ wangcx
}

$$
\epsilon_{x}=C_{q} \gamma^{2} \frac{\left\langle\mathcal{H} /|\rho|^{3}\right\rangle}{J_{x}\left\langle 1 / \rho^{2}\right\rangle}, \quad \sigma_{\delta}^{2}=C_{q} \gamma^{2} \frac{\left\langle 1 /|\rho|^{3}\right\rangle}{J_{E}\left\langle 1 / \rho^{2}\right\rangle},
$$

where $C_{q}=(55 / 32 \sqrt{3})(\hbar / m c)=3.83 \times 10^{-13} \mathrm{~m}$ for electrons; $\gamma$ is the Lorentz factor of beam energy (which will be used only in the form $C_{q} \gamma^{2}$, and not be confused with the Courant-Snyder parameter); $J_{x}$ and $J_{E}$ are the horizontal and longitudinal damping partition numbers, respectively; $\rho$ is the dipole bending radius; the brackets $\langle\cdots\rangle$ indicate averaging over the bending magnets; and the dispersion action

$$
\mathcal{H}=\gamma \eta^{2}+2 \alpha \eta \eta^{\prime}+\beta \eta^{\prime 2},
$$

where $\beta, \alpha$, and $\gamma$ are the Courant-Snyder (C-S) parameters of horizontal betatron motion, $\eta$ and $\eta^{\prime}$ are the dispersion and its derivative, respectively. Since dipole contributions to the emittance are accumulative, it is desirable to have all dipoles contribute as little as possible. Thus, the minimization over a ring reduces to minimization over individual dipoles, and the whole ring simply consists of the same optimal dipole configuration (except for the need to match the end dipoles with those inside a multibend achromat [4]). Thus, this paper will focus on minimization for an individual dipole with or without bending radius variation.

This paper is organized as follows: In Sec. II, we first treat the classical uniform dipole case in order to introduce our new formalism and help readers to appreciate its elegance and simplicity. In addition to the basic theory presented in Sec. II A, dipoles with transverse gradient are treated as a concrete example in Sec. II B, followed by a simple computation of nonoptimal-lattice effects in Sec. II C. In Sec. III, we generalize our theory to arbitrary dipoles with emphasis on nonuniform dipoles with bending radius variation. Section III A presents the general theory and deduces a couple of basic properties. Section III B presents minimization of the effective emittance. Section IIIC gives a concrete example of nonuniform 
dipole made of three uniform pieces. Section III D briefly examines the nonlinear magnetic field caused by a nonuniform dipole. In Sec. IV, we investigate the parameter dependence of the effective emittance on the damping partition numbers and nonuniform dipoles. A brief conclusion is given in Sec. V. Most of the derivations, especially the basic theory, are presented in sufficient detail and should be easy to follow.

\section{MINIMUM EMITTANCE WITH UNIFORM DIPOLES}

Usually the bending field (and radius $\rho$ ) in dipoles can be assumed constant and are often the same in a whole ring (isomagnetic). Thus, the emittance depends on the lattice through $\langle\mathcal{H}\rangle$. We will consider this well-studied uniform dipole case first.

\section{A. Simple formulas for emittance minimization}

Computation of $\langle\mathcal{H}\rangle$ is straightforward [13] but can be tedious, and its minimization can be obscured by the algebra. Here we derive simple formulas to make the algebra more transparent. Geometrically, $\mathcal{H}$ is the length square of the dispersion vector in the Floquet space, i.e.,

$$
\begin{aligned}
\mathcal{H} & =\|\tilde{\boldsymbol{\eta}}\|^{2}=\operatorname{Tr}\left(\tilde{\boldsymbol{\eta}} \tilde{\boldsymbol{\eta}}^{T}\right)=\operatorname{Tr}\left(\mathcal{A} \boldsymbol{\eta} \boldsymbol{\eta}^{T} \mathcal{A}^{T}\right) \\
& =\operatorname{Tr}\left(\boldsymbol{\eta} \boldsymbol{\eta}^{T} \sigma^{+}\right),
\end{aligned}
$$

where $\boldsymbol{\eta}$ and $\tilde{\boldsymbol{\eta}}=\mathcal{A} \boldsymbol{\eta}$ are the dispersion vectors in phase space and Floquet space, respectively. $\sigma^{+} \equiv-J \sigma^{T} J$ is the symplectic conjugate of the normalized beam matrix $\sigma$. More explicitly,

$$
\begin{gathered}
\tilde{\boldsymbol{\eta}}=\left[\begin{array}{c}
\tilde{\eta} \\
\tilde{\eta}^{\prime}
\end{array}\right]=\mathcal{A}\left[\begin{array}{c}
\eta \\
\eta^{\prime}
\end{array}\right], \quad \mathcal{A}=\left[\begin{array}{cc}
1 / \sqrt{\beta} & 0 \\
\alpha / \sqrt{\beta} & \sqrt{\beta}
\end{array}\right], \\
\mathcal{A}^{T} \mathcal{A}=\sigma^{+},
\end{gathered}
$$

and

$$
\begin{gathered}
\sigma^{+}=\left[\begin{array}{cc}
\gamma & \alpha \\
\alpha & \beta
\end{array}\right], \quad \sigma=\left[\begin{array}{cc}
\beta & -\alpha \\
-\alpha & \gamma
\end{array}\right], \\
J=\left[\begin{array}{cc}
0 & 1 \\
-1 & 0
\end{array}\right] .
\end{gathered}
$$

The dispersion vector propagates within a dipole as

$$
\boldsymbol{\eta}(s)=M(s) \boldsymbol{\eta}_{0}+\boldsymbol{\xi}(s)=M(s)\left[\boldsymbol{\eta}_{0}+\hat{\boldsymbol{\xi}}(s)\right],
$$

where $\hat{\boldsymbol{\xi}}(s) \equiv M^{-1} \boldsymbol{\xi}$; the transfer matrix $M(s)$ and the dispersion generating vector $\boldsymbol{\xi}(s)$ are determined by the dipole magnet. Hereafter, subscript 0 indicates values at the dipole entrance. Inserting Eq. (6) into Eq. (3) yields

$$
\mathcal{H}=\operatorname{Tr}\left\{\left(\boldsymbol{\eta}_{0}+\hat{\boldsymbol{\xi}}\right)\left(\boldsymbol{\eta}_{0}+\hat{\boldsymbol{\xi}}\right)^{T} M^{T} \sigma^{+} M\right\}
$$

Using $\sigma(s)=M \sigma_{0} M^{T}$ and the symplectic condition $M M^{+}=M^{+} M=1$, we have

$$
\begin{aligned}
\mathcal{H} & =\operatorname{Tr}\left\{\left(\boldsymbol{\eta}_{0}+\hat{\boldsymbol{\xi}}\right)\left(\boldsymbol{\eta}_{0}+\hat{\boldsymbol{\xi}}\right)^{T} \sigma_{0}^{+}\right\} \\
& =\operatorname{Tr}\left\{\left(\boldsymbol{\eta}_{0} \boldsymbol{\eta}_{0}^{T}+\boldsymbol{\eta}_{0} \hat{\boldsymbol{\xi}}^{T}+\hat{\boldsymbol{\xi}} \boldsymbol{\eta}_{0}^{T}+\hat{\boldsymbol{\xi}} \hat{\boldsymbol{\xi}}^{T}\right) \sigma_{0}^{+}\right\}
\end{aligned}
$$

Note that $\boldsymbol{\eta}_{0} \hat{\boldsymbol{\xi}}^{T}$ and $\hat{\boldsymbol{\xi}} \boldsymbol{\eta}_{0}^{T}$ contribute equally to $\mathcal{H}$, although we kept this symmetric form for other purposes. This simple expression nicely separates the initial values and $s$-dependent quantities determined by the magnet via $\hat{\boldsymbol{\xi}}(s)$. Furthermore, integration can be carried through the trace operation as well as initial conditions. Therefore, the key quantity $\langle\mathcal{H}\rangle$ is given by the simple expression

$$
\langle\mathcal{H}\rangle=\operatorname{Tr}\left(\mathcal{A}_{0} G_{0} \mathcal{A}_{0}^{T}\right)=\operatorname{Tr}\left(G_{0} \sigma_{0}^{+}\right)
$$

with

$$
\begin{aligned}
G_{0} & \equiv \boldsymbol{\eta}_{0} \boldsymbol{\eta}_{0}^{T}+\boldsymbol{\eta}_{0}\langle\hat{\boldsymbol{\xi}}\rangle^{T}+\langle\hat{\boldsymbol{\xi}}\rangle \boldsymbol{\eta}_{0}^{T}+\left\langle\hat{\boldsymbol{\xi}} \hat{\boldsymbol{\xi}}^{T}\right\rangle \\
& =\left(\boldsymbol{\eta}_{0}+\langle\hat{\boldsymbol{\xi}}\rangle\right)\left(\boldsymbol{\eta}_{0}+\langle\hat{\boldsymbol{\xi}}\rangle\right)^{T}+\left\langle\hat{\boldsymbol{\xi}} \hat{\xi}^{T}\right\rangle-\langle\hat{\boldsymbol{\xi}}\rangle\langle\hat{\boldsymbol{\xi}}\rangle^{T} .
\end{aligned}
$$

To minimize $\langle\mathcal{H}\rangle$, we consider the Schur decomposition of the real symmetric matrix $\mathcal{A}_{0} G_{0} \mathcal{A}_{0}^{T}=$ $V \operatorname{diag}\left(\lambda_{1}, \lambda_{2}\right) V^{-1}$ with some orthogonal matrix $V$, where the eigenvalues $\lambda_{1}$ and $\lambda_{2}$ must be real. Thus, $\langle\mathcal{H}\rangle=\lambda_{1}+$ $\lambda_{2}$ and $\left|G_{0}\right|=\lambda_{1} \lambda_{2}$ [14]. Since $\langle\mathcal{H}\rangle \geq 0$, if assuming $\left|G_{0}\right|>0$, then the eigenvalues are all positive real numbers that must satisfy $\lambda_{1}+\lambda_{2} \geq 2 \sqrt{\lambda_{1} \lambda_{2}}$ with equality holds only when $\lambda_{1}=\lambda_{2}$. Therefore,

$$
\langle\mathcal{H}\rangle \geq 2 \sqrt{\left|G_{0}\right|}, \quad \text { equality holds iff } \sigma_{0}=\frac{G_{0}}{\sqrt{\left|G_{0}\right|}} .
$$

The optimal C-S parameters depend on the initial dispersion $\boldsymbol{\eta}_{0}$ explicitly via $G_{0} / \sqrt{\left|G_{0}\right|}$. For achromatic lattices, $\boldsymbol{\eta}_{0}=0, G_{0}=\left\langle\hat{\xi} \hat{\xi}^{T}\right\rangle$, and

$$
\langle\mathcal{H}\rangle_{\min }^{\boldsymbol{\eta}_{0}=0}=2 \sqrt{\left|\left\langle\hat{\boldsymbol{\xi}} \hat{\boldsymbol{\xi}}^{T}\right\rangle\right|} .
$$

Since $\left(\boldsymbol{\eta}_{0}+\langle\hat{\boldsymbol{\xi}}\rangle\right)\left(\boldsymbol{\eta}_{0}+\langle\hat{\boldsymbol{\xi}}\rangle\right)^{T}$ contributes a positive term to $\langle\mathcal{H}\rangle$, the condition to further minimize $\left|G_{0}\right|$ through $\boldsymbol{\eta}_{0}$ is clearly given by

$$
\boldsymbol{\eta}_{0}=-\langle\hat{\boldsymbol{\xi}}\rangle,
$$

which yields the absolute minimum

$$
\langle\mathcal{H}\rangle_{\min }^{\mathrm{TME}}=2 \sqrt{\left|\left\langle\hat{\boldsymbol{\xi}} \hat{\boldsymbol{\xi}}^{T}\right\rangle-\langle\hat{\boldsymbol{\xi}}\rangle\langle\hat{\boldsymbol{\xi}}\rangle^{T}\right|}
$$

This gives the theoretical minimum emittance and is thus labeled TME.

One may choose other locations instead of the dipole entrance for initial conditions. Of particular interest is the center of the dipole. Using the subscript " $c$ " to indicate values at the dipole center, Eq. (9) reduces to

$$
\langle\mathcal{H}\rangle=\operatorname{Tr}\left(G_{c} \sigma_{c}^{+}\right),
$$

where 


$$
G_{c}=\left[\begin{array}{cc}
\eta_{c}^{2}+2\langle\hat{\xi}\rangle_{c} \eta_{c}+\left\langle\hat{\xi}^{2}\right\rangle_{c} & \left(\eta_{c}+\langle\hat{\xi}\rangle_{c}\right) \eta_{c}^{\prime} \\
\left(\eta_{c}+\langle\hat{\xi}\rangle_{c}\right) \eta_{c}^{\prime} & \eta_{c}^{\prime 2}+\left\langle\hat{\xi}^{\prime 2}\right\rangle_{c}
\end{array}\right]
$$

Here, $\langle\cdots\rangle_{c}$ means averaging around the dipole center, i.e., from $-\theta / 2$ to $\theta / 2$, where $\theta$ is the total bending angle. Note that $\left\langle\hat{\xi}^{\prime}\right\rangle_{c}$ and $\left\langle\hat{\xi} \hat{\xi}^{\prime}\right\rangle_{c}$ vanish due to symmetry. As before, the minimum value is given by $2 \sqrt{\left|G_{c}\right|}$, which yields

$$
\langle\mathcal{H}\rangle_{\min }=2 \sqrt{\left(\left\langle\hat{\xi}^{2}\right\rangle_{c}-\langle\hat{\xi}\rangle_{c}^{2}\right)\left(\eta_{c}^{\prime 2}+\left\langle\hat{\xi}^{\prime 2}\right\rangle_{c}\right)+\left(\eta_{c}+\langle\hat{\xi}\rangle_{c}\right)^{2}\left\langle\hat{\xi}^{\prime 2}\right\rangle_{c}}
$$

Obviously, the absolute minimum $2 \sqrt{\left(\left\langle\hat{\xi}^{2}\right\rangle_{c}-\langle\hat{\xi}\rangle_{c}^{2}\right)\left\langle\hat{\xi}^{\prime 2}\right\rangle_{c}}$ is reached when $\eta_{c}^{\prime}=0$ and $\eta_{c}=-\langle\hat{\xi}\rangle_{c}$, which leads to $\alpha_{c}=0$ and $\beta_{c}=\sqrt{\left(\left\langle\hat{\xi}^{2}\right\rangle_{c}-\langle\hat{\xi}\rangle_{c}^{2}\right) /\left\langle\hat{\xi}^{\prime 2}\right\rangle_{c}}$ from Eqs. (16) and (11). Thus, both beta function and dispersion must be symmetric about the dipole center in order to reach the absolute minimum emittance. However, to maintain the achromatic condition, $\boldsymbol{\eta}_{c}=-\hat{\boldsymbol{\xi}}(-\theta / 2)$ is required from Eq. (6).

This simple treatment and resulting formulas offer a new viewpoint to the theory of minimum emittance in storage rings. Note that no approximation (such as parabolic $s$ dependence of beta and dispersion functions, or small bending angle) has been used in driving this general result. Generalization to include nonuniform dipoles and minimization of the effective emittance for light sources will be given in Sec. III.

\section{B. Minimum emittance of transverse gradient dipoles}

For a combined-function sector dipole of constant bending radius $\rho$ and bending angle $\theta$ with a transverse gradient $K=(1 / B \rho) \partial B_{y} / \partial x$, the transfer matrix reads

$$
\begin{aligned}
& M=\left[\begin{array}{cc}
\cos (k \theta) & \frac{\rho}{k} \sin (k \theta) \\
-\frac{k}{\rho} \sin (k \theta) & \cos (k \theta)
\end{array}\right], \\
& \boldsymbol{\xi}=\left[\begin{array}{c}
\frac{\rho}{k^{2}}(1-\cos (k \theta)) \\
\frac{1}{k} \sin (k \theta)
\end{array}\right],
\end{aligned}
$$

and

$$
\hat{\boldsymbol{\xi}}=M^{-1} \boldsymbol{\xi}=\left[\begin{array}{c}
\rho(\cos (k \theta)-1) / k^{2} \\
\sin (k \theta) / k
\end{array}\right],
$$

where $k=\sqrt{1+K \rho^{2}}$. After averaging with respect to the dipole center, we have

$$
\begin{gathered}
\langle\hat{\xi}\rangle_{c}=\frac{2 \rho}{\theta k^{3}}\left(\sin \frac{k \theta}{2}-\frac{k \theta}{2}\right), \\
\left\langle\hat{\xi}^{2}\right\rangle_{c}=\frac{\rho^{2}}{2 \theta k^{5}}\left(\sin (k \theta)-8 \sin \frac{k \theta}{2}+3 k \theta\right), \\
\left\langle\hat{\xi}^{\prime 2}\right\rangle_{c}=\frac{1}{2 \theta k^{3}}[k \theta-\sin (k \theta)] .
\end{gathered}
$$

Inserting these into Eq. (17) gives exact expressions for the minimum emittances. For most practical applications, it is sufficient to use the following small-angle approximations:

$$
\langle\mathcal{H}\rangle_{\min }^{\boldsymbol{\eta}_{0}=0}=\frac{\rho \theta^{3}}{4 \sqrt{15}}-\frac{11 \rho \theta^{5}\left(1+K \rho^{2}\right)}{840 \sqrt{15}}+O\left(\theta^{7}\right)
$$

and

$$
\langle\mathcal{H}\rangle_{\min }^{\mathrm{TME}}=\frac{\rho \theta^{3}}{12 \sqrt{15}}-\frac{\rho \theta^{5}\left(1+K \rho^{2}\right)}{280 \sqrt{15}}+O\left(\theta^{7}\right)
$$

Boundary parameters of a dipole magnet, such as pole-face rotation angles and fringe-field integrals, will not affect these results because they contribute only thin quadrupoles at the boundaries. Setting $K=0$ gives the well-known result for the theoretical minimum emittances with and without achromatic conditions. The gradient contributes only a factor $1+K \rho^{2}$ in the small correction term. Thus, it can affect $\langle\mathcal{H}\rangle_{\min }$ appreciably only when $K L^{2}$ is much larger than 1 , where $L$ is the dipole length. However, the gradient is more effective at increasing the damping partition number $J_{x}$ and thus has been used to lower the emittance in some machines. See Sec. IVA for a detailed discussion.

For completeness, Table I lists the optimal lattice parameters at the entrance and the center of a dipole under small-angle approximation. Exact expressions are straightforward to compute using the results in the previous section. Note that the optimal parameters at the entrance may be affected by the dipole edge effects.

TABLE I. Optimal lattice parameters at the entrance and the center of a dipole under achromatic and TME conditions.

\begin{tabular}{lcc}
\hline \hline & Achromatic & TME \\
\hline$\beta_{0}$ & $\frac{6}{\sqrt{15}} \rho \theta-\frac{2}{5 \sqrt{15}} \rho k^{2} \theta^{3}+\cdots$ & $\frac{8}{\sqrt{15}} \rho \theta-\frac{4}{5 \sqrt{15}} \rho k^{2} \theta^{3}+\cdots$ \\
$\alpha_{0}$ & $\sqrt{15}-\frac{20}{7 \sqrt{15}} k^{2} \theta^{2}+\cdots$ & $\sqrt{15}-\frac{12}{7 \sqrt{15}} k^{2} \theta^{2}+\cdots$ \\
$\eta_{0}$ & 0 & $\frac{1}{6} \rho \theta^{2}-\frac{1}{120} \rho k^{2} \theta^{4}+\cdots$ \\
$\eta_{0}^{\prime}$ & 0 & $-\frac{1}{2} \theta+\frac{1}{24} k^{2} \theta^{3}+\cdots$ \\
\hline$\beta_{c}$ & $\frac{1}{\sqrt{15}} \rho \theta+\frac{1}{210 \sqrt{15}} \rho k^{2} \theta^{3}+\cdots$ & $\frac{1}{2 \sqrt{15}} \rho \theta+\frac{1}{280 \sqrt{15}} \rho k^{2} \theta^{3}+\cdots$ \\
$\alpha_{c}$ & $-\frac{5}{\sqrt{15}}+\frac{1}{14 \sqrt{15}} k^{2} \theta^{2}+\cdots$ & 0 \\
$\eta_{c}$ & $\frac{1}{8} \rho \theta^{2}-\frac{1}{38} \rho k^{2} \theta^{4}+\cdots$ & $\frac{1}{24} \rho \theta^{2}-\frac{1}{1920} \rho k^{2} \theta^{4}+\cdots$ \\
$\eta_{c}^{\prime}$ & $\frac{1}{2} \theta-\frac{1}{48} k^{2} \theta^{3}+\cdots$ & 0 \\
\hline \hline
\end{tabular}




\section{Effects of nonoptimal-lattice parameters}

Even in storage rings designed for low emittance, often the optimal condition $\sigma_{0}=G_{0} / \sqrt{\left|G_{0}\right|}$ in Eq. (11) for emittance minimization cannot be satisfied. Assuming $\tilde{\beta}=\beta_{0} / \beta_{0}^{\mathrm{opt}}$ and $\tilde{\alpha}=\alpha_{0} / \alpha_{0}^{\mathrm{opt}}$, the resulting emittance degradation can be calculated simply from Eqs. (9) and (11) as

$$
\begin{aligned}
\frac{\langle\mathcal{H}\rangle}{\langle\mathcal{H}\rangle_{\min }} & =\frac{1}{2} \operatorname{Tr}\left\{\sigma_{0}^{\mathrm{opt}} \sigma_{0}^{+}\right\}=\frac{1}{2} \operatorname{Tr}\left\{\left(\sigma_{0}^{\mathrm{opt}}\right)^{+} \sigma_{0}\right\} \\
& =\frac{1}{2}\left(\tilde{\beta}+\frac{1}{\tilde{\beta}}\right)+\frac{1}{2}\left(\sqrt{\tilde{\beta}}-\frac{\tilde{\alpha}}{\sqrt{\tilde{\beta}}}\right)^{2}\left(\alpha_{0}^{\mathrm{opt}}\right)^{2},
\end{aligned}
$$

where the optimal $\alpha$ at dipole entrance is given by $\alpha_{0}^{\text {opt }} \simeq$ $\sqrt{15}$ for both the achromatic and nonachromatic cases. A contour plot of this ratio is shown in Fig. 1. The degradation is minimal along the diagonal because of the removal of the second term in Eq. (25) when $\tilde{\alpha}=\tilde{\beta}$. This is a nice clean formula for evaluating the sensitivity of emittance on the C-S parameters at the dipole entrance. For a given $\tilde{\beta}$, the minimum emittance degradation is simply $(\tilde{\beta}+$ $1 / \tilde{\beta}) / 2$, which is much smaller than the degradation resulting from keeping $\alpha_{0}$ at its optimal value (as often considered).

The optimal dispersion is given by $\boldsymbol{\eta}_{0}=q\langle\hat{\boldsymbol{\xi}}\rangle$ with $q=$ 0 and -1 for achromatic and TME lattices, respectively. For nonoptimal dispersion but still parallel to the vector $\langle\hat{\boldsymbol{\xi}}\rangle$, its effect can be computed by

$$
\begin{aligned}
\langle\mathcal{H}\rangle & =2 \sqrt{|A+\tilde{q} B|}=2 \sqrt{|A|\left|I+\tilde{q} A^{-1} B\right|} \\
& =2 \sqrt{|A|\left|I+\tilde{q} A^{+} B /\right| A||} \\
& =2 \sqrt{|A|\left(1+\tilde{q} \operatorname{Tr}\left(A^{+} B\right) /|A|\right)} .
\end{aligned}
$$

Here $A=\left\langle\hat{\boldsymbol{\xi}} \hat{\boldsymbol{\xi}}^{T}\right\rangle, B=\langle\hat{\boldsymbol{\xi}}\rangle\langle\hat{\boldsymbol{\xi}}\rangle^{T}$, and $\tilde{q}=q^{2}+2 q$. In the last step we have used $\left|A^{+} B\right| \propto|B|=0$. For uniform di-

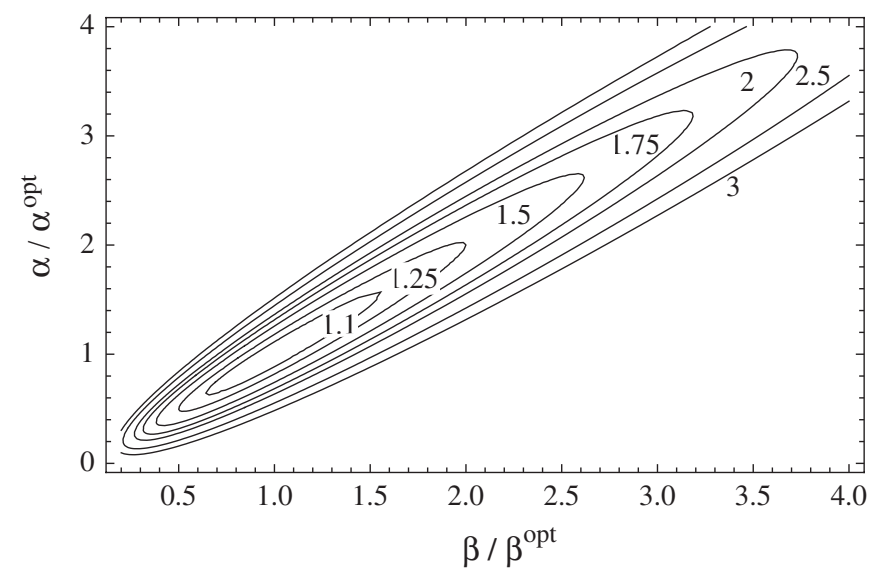

FIG. 1. Contours of emittance degradation due to nonoptimal Courant-Snyder parameters at the dipole entrance. poles, $2 \sqrt{|A|}$ is given by Eq. (23) and $\operatorname{Tr}\left(A^{+} B\right) /|A| \simeq 8 / 9$ from

$$
\operatorname{Tr}\left(\langle\hat{\boldsymbol{\xi}}\rangle\langle\hat{\boldsymbol{\xi}}\rangle^{T}\left\langle\hat{\boldsymbol{\xi}} \hat{\boldsymbol{\xi}}^{T}\right\rangle^{+}\right) /\left|\left\langle\hat{\boldsymbol{\xi}} \hat{\boldsymbol{\xi}}^{T}\right\rangle\right|=\frac{8}{9}-\frac{2 k^{2} \theta^{2}}{945}+O\left(\theta^{4}\right) .
$$

Thus, the effect of nonoptimal $q$ can be obtained simply from $\sqrt{1+(8 / 9)\left(q^{2}+2 q\right)}$.

\section{MINIMUM EMITTANCE WITH NONUNIFORM DIPOLES}

Nonuniform dipoles with longitudinally varying field have been investigated for reducing the emittance below the well-known theoretical minimum in uniform dipoles. Such a possibility was first considered by Wrulich in 1992 as claimed in [11]. Here we present a general formalism for emittance minimization with nonuniform dipole, without giving an optimal field profile which may depend on practical limitations.

\section{A. Emittance minimization with nonconstant $\rho(s)$}

To reduce emittance below the theoretical minimum emittance given above, one has to consider nonuniform dipoles with an $s$-dependent bending radius. Since $\mathcal{H}$ is always positive, $\left\langle\mathcal{H} /|\rho|^{3}\right\rangle$ may become smaller when $\rho(s)$ is tailored to make $\mathcal{H} /|\rho|^{3}$ more uniform, while nonuniform $\rho$ tends to make the denominator $\left\langle 1 / \rho^{2}\right\rangle$ larger. The details are more complicated. Because Eq. (8) holds for any dipole as long as its nonlinear effects are negligible in dispersion propagation, which we will assume, it is easy to see that the emittance minimization theory in Sec. II A can be extended to nonuniform dipoles by minimizing

$$
\langle\langle\mathcal{H}\rangle\rangle \equiv \frac{\left\langle\mathcal{H} /|\rho|^{3}\right\rangle}{\left\langle 1 / \rho^{2}\right\rangle}=\operatorname{Tr}\left(G_{0} \sigma_{0}^{+}\right)
$$

where

$$
G_{0} \equiv \check{\rho} \boldsymbol{\eta}_{0} \boldsymbol{\eta}_{0}^{T}+\boldsymbol{\eta}_{0}\langle\langle\hat{\boldsymbol{\xi}}\rangle\rangle^{T}+\langle\langle\hat{\boldsymbol{\xi}}\rangle\rangle \boldsymbol{\eta}_{0}^{T}+\left\langle\left\langle\hat{\boldsymbol{\xi}} \hat{\boldsymbol{\xi}}^{T}\right\rangle\right\rangle,
$$

and

$$
\langle\langle v\rangle\rangle \equiv \frac{\left\langle v /|\rho|^{3}\right\rangle}{\left\langle 1 / \rho^{2}\right\rangle} \quad \text { and } \quad \check{\rho} \equiv\langle\langle 1\rangle\rangle=\frac{\left\langle 1 /|\rho|^{3}\right\rangle}{\left\langle 1 / \rho^{2}\right\rangle} .
$$

Thus, as before, the minimum emittance is given by $\langle\langle\mathcal{H}\rangle\rangle_{\min }=2 \sqrt{\left|G_{0}\right|}$ with the optimal C-S parameters $\sigma_{0}=G_{0} / \sqrt{\left|G_{0}\right|}$ at the dipole entrance. Let

$$
\boldsymbol{\eta}_{0}=q\langle\langle\hat{\boldsymbol{\xi}}\rangle\rangle / \check{\rho}
$$

and

$$
G_{0}(q)=\left\langle\left\langle\hat{\boldsymbol{\xi}} \hat{\boldsymbol{\xi}}^{T}\right\rangle\right\rangle+q\langle\langle\hat{\boldsymbol{\xi}}\rangle\rangle\langle\langle\hat{\boldsymbol{\xi}}\rangle\rangle^{T} / \check{\rho} \equiv A+q B,
$$

then the optimal $\boldsymbol{\eta}_{0}^{\text {opt }}, \sigma_{0}^{\text {opt }}$, and $G_{0}^{\text {opt }}$ are given by $q^{\text {opt }}=0$ for achromatic lattices and $q^{\mathrm{opt}}=-1$ for TME lattices. 
Later we will see that certain value in between is required to minimize the effective emittance for light sources. Be careful that Eq. (29) reduces to $G_{0}\left(q^{2}+2 q\right)$ instead of $G_{0}(q)$ under the condition in Eq. (30). We further introduce a parameter

$$
c \equiv \operatorname{Tr}\left(A B^{+}\right) /|A|=-\operatorname{Tr}(J A J B) /|A| .
$$

Explicitly $\quad \operatorname{Tr}\left(A B^{+}\right)=\left(A_{1} d_{2}^{2}-2 A_{2} d_{1} d_{2}+A_{3} d_{1}^{2}\right) / \check{\rho}$, where $A_{1}, A_{2}$, and $A_{3}$ are the $(1,1),(1,2)$, and $(2,2)$ elements of the matrix $\left\langle\left\langle\hat{\boldsymbol{\xi}} \hat{\boldsymbol{\xi}}^{T}\right\rangle\right\rangle$ and $\left[d_{1}, d_{2}\right]^{T}=\langle\langle\hat{\boldsymbol{\xi}}\rangle\rangle$. From Eq. (26), we have

$$
\langle\langle\mathcal{H}\rangle\rangle_{\min }=2 \sqrt{\left|G_{0}^{\mathrm{opt}}\right|}=2 \sqrt{|A|} \sqrt{1+q^{\mathrm{opt}} c} .
$$

Since $\langle\langle\mathcal{H}\rangle\rangle_{\min }^{\mathrm{TME}} \propto \sqrt{1-c}$, the incoherent motion excited in dipoles as measured by the emittance may approach zero as $c$ approaches 1 . Thus we will refer $c$ as coherent parameter or $c$ parameter of a dipole. For a uniform dipole, $c \simeq 8 / 9$. Later we will see that $c$ may approach 1 for nonuniform dipoles. Note that $|A|, c$, and $\langle\langle\mathcal{H}\rangle\rangle_{\min }$ are completely determined by the local linear beam transport inside a dipole.

A couple of general conclusions can be deduced without detailed knowledge of $\rho(s)$. First of all, unlike in uniform dipoles, no absolute minimum emittance exists if $\rho(s)$ is not constrained. This seems obvious if we allow $\rho(s) \rightarrow 0$ at a point, in which case $\langle\langle x\rangle\rangle \rightarrow x$ (at the singular point) $\check{\rho}$ and thus $\left.\left\langle\left\langle\hat{\boldsymbol{\xi}} \hat{\boldsymbol{\xi}}^{T}\right\rangle\right\rangle-\langle\langle\hat{\boldsymbol{\xi}}\rangle\rangle\langle\hat{\boldsymbol{\xi}}\rangle\right\rangle^{T} / \check{\rho} \rightarrow 0$. In fact, it is more subtle because both $\left\langle\left\langle\hat{\boldsymbol{\xi}} \hat{\boldsymbol{\xi}}^{T}\right\rangle\right\rangle$ and $\langle\langle\hat{\boldsymbol{\xi}}\rangle\rangle\langle\langle\hat{\boldsymbol{\xi}}\rangle\rangle^{T} / \check{\rho}$ approach infinity as their difference approaches zero. Thus the outcome depends on more details. If we assume $\rho(s)=\rho_{a}+$ $\left(\rho_{a}^{\prime \prime} / 2\right) d s^{2}$ and $\xi(s)=\xi_{a}+\xi_{a}^{\prime} d s+\left(\xi_{a}^{\prime \prime} / 2\right) d s^{2}$ close to the singularity, then it is straightforward to carry out the integrations over a small neighborhood around the singularity. It can be shown that, after taking the limit $\rho_{a} \rightarrow 0$, the matrix $\left\langle\left\langle\hat{\boldsymbol{\xi}} \hat{\boldsymbol{\xi}}^{T}\right\rangle\right\rangle-\langle\langle\hat{\boldsymbol{\xi}}\rangle\rangle\langle\langle\hat{\boldsymbol{\xi}}\rangle\rangle^{T} / \check{\rho}$ reduces to $\left[\xi_{a}^{\prime 2}, \xi_{a}^{\prime} \xi_{a}^{\prime \prime} ; \xi_{a}^{\prime} \xi_{a}^{\prime \prime}, \xi_{a}^{\prime \prime 2}\right] / 2 \rho_{a}^{\prime \prime}$, whose determinant is zero. Thus, in principle, the emittance can approach zero. On the other hand, later we will show that, in some cases, there is an absolute minimum even when the field goes to infinity.

Another general conclusion is that $\left|G^{\text {opt }}\right|$ and thus the minimum emittance is scale invariant. In other words it is invariant under arbitrary scaling of $\rho(s)$, i.e., only the relative profile of $\rho(s)$ matters. To see this, we recall that the linear Hamiltonian of a sector dipole without a transverse gradient reads $H=\left(p_{x}^{2}+p_{y}^{2}\right) / 2+x^{2} / 2 \rho(s)^{2}-$ $x \delta / \rho(s)$, where $p_{x}, p_{y}, x$ are the transverse dynamical variables and $\delta$ is the relative energy deviation. If all spatial quantities such as $x, s$, as well as $\rho(s)$ are scaled by the same constant $\lambda$, the Hamiltonian $H$ and corresponding dynamics will remain the same. Thus $\hat{\xi} \propto \lambda$ and $\hat{\xi}^{\prime}$ is unchanged. Put these into Eq. (31), and it is clear that $\left|G^{\text {opt }}\right|$ is independent of the scaling constant $\lambda$. For gradient dipoles, this is not exactly true but the gradient effects are expected to be small as in a uniform gradient dipole. For uniform dipoles, scale invariant leads to the well-known fact that the minimum emittance does not depend on the bending radius. It should be noted that, although the minimum emittance is scale invariant, the optimal C-S parameters and dispersion do depend on the magnitude of bending radius ( $\beta^{\text {opt }}$ and $\eta^{\text {opt }}$ are proportional to $\lambda$, while $\alpha^{\mathrm{opt}}$ and $\eta^{\text {opt }}$ are independent of $\lambda$ ).

\section{B. Minimization of the effective emittance}

It was pointed out [5] that the rms emittance including the effects of energy spread, referred to as the effective emittance, should be minimized for light-source storage rings. The effective emittance can be calculated by

$$
\begin{aligned}
\epsilon_{\mathrm{eff}}^{2} & \equiv\left\langle(x+\eta \delta)^{2}\right\rangle\left\langle\left(x^{\prime}+\eta^{\prime} \delta\right)^{2}\right\rangle-\left\langle(x+\eta \delta)\left(x^{\prime}+\eta^{\prime} \delta\right)\right\rangle^{2} \\
& =\left|\left\langle(\boldsymbol{x}+\boldsymbol{\eta} \delta)(\boldsymbol{x}+\boldsymbol{\eta} \delta)^{T}\right\rangle\right|=\left|\epsilon_{x} \sigma+\boldsymbol{\eta} \boldsymbol{\eta}^{T} \sigma_{\delta}^{2}\right| \\
& =\epsilon_{x}^{2}\left|I+\left(\sigma_{\delta}^{2} / \boldsymbol{\epsilon}_{x}\right) \boldsymbol{\eta} \boldsymbol{\eta}^{T} \sigma^{+}\right| \\
& =\boldsymbol{\epsilon}_{x}^{2}\left[1+\left(\sigma_{\delta}^{2} / \boldsymbol{\epsilon}_{x}\right) \operatorname{Tr}\left(\boldsymbol{\eta} \boldsymbol{\eta}^{T} \sigma^{+}\right)\right] \\
& =\boldsymbol{\epsilon}_{x}\left(\boldsymbol{\epsilon}_{x}+\mathcal{H}_{\mathrm{ID}} \sigma_{\delta}^{2}\right),
\end{aligned}
$$

where the average is over phase space instead of position $s$ as used elsewhere in this paper. In the second to the last step, we have used the fact $\left|\boldsymbol{\eta} \boldsymbol{\eta}^{T} \sigma^{+}\right|=0$. The dispersion action $\mathcal{H}_{\mathrm{ID}}$ can be computed anywhere in a straight section because it is invariant outside bending magnets. We will take the end of a straight section and the entrance of a dipole (marked with the subscript " 0 ") for our computation. Taking Eqs. (1) and (28) into account, the effective emittance becomes $\epsilon_{\text {eff }}^{2}=\left(C_{q} \gamma^{2} / J_{x}\right)^{2} F$, where

$$
\begin{aligned}
F & =\langle\langle\mathcal{H}\rangle\rangle\left[\langle\langle\mathcal{H}\rangle\rangle+\left(J_{x} / J_{E}\right) \check{\rho} \mathcal{H}_{0}\right] \\
& =\operatorname{Tr}\left(G_{0} \sigma_{0}^{+}\right) \operatorname{Tr}\left(\left[G_{0}+\tau \check{\rho} \boldsymbol{\eta}_{0} \boldsymbol{\eta}_{0}^{T}\right] \sigma_{0}^{+}\right)
\end{aligned}
$$

with

$$
\tau \equiv J_{x} / J_{E}
$$

Minimization of $F$ is much more involved due to the product of matrix traces and higher order polynomials.

Inserting Eq. (29) into $F$ and computing its change $\Delta F$ due to an initial dispersion variation $\Delta \boldsymbol{\eta}_{0}$ yields

$$
\begin{aligned}
\Delta F= & \operatorname{Tr}\left[2 \Delta \boldsymbol{\eta}_{0}\left(\check{\rho} \boldsymbol{\eta}_{0}+\langle\langle\hat{\boldsymbol{\xi}}\rangle\rangle\right)^{T} \sigma_{0}^{+}\right] \operatorname{Tr}\left(\tilde{G}_{0} \sigma_{0}^{+}\right) \\
& +\operatorname{Tr}\left\{2 \Delta \boldsymbol{\eta}_{0}\left[(1+\tau) \check{\rho} \boldsymbol{\eta}_{0}+\langle\langle\hat{\boldsymbol{\xi}}\rangle\rangle\right]^{T} \sigma_{0}^{+}\right\} \operatorname{Tr}\left(G_{0} \sigma_{0}^{+}\right),
\end{aligned}
$$

where $\tilde{G}_{0} \equiv G_{0}+\tau \check{\rho} \boldsymbol{\eta}_{0} \boldsymbol{\eta}_{0}^{T}$. Notice that, when $\boldsymbol{\eta}_{0} \propto\langle\langle\hat{\boldsymbol{\xi}}\rangle\rangle$, the two traces containing $\Delta \boldsymbol{\eta}_{0}$ becomes proportional to each other, and thus their ratio can be used to solve $\Delta F=0$ for all $\Delta \boldsymbol{\eta}_{0}$, which is required to minimize $F$. Therefore, we insert Eq. (30) into $\Delta F=0$ to get the equation for the optimal $q$ as

$$
(q+1) \operatorname{Tr}\left(\tilde{G}_{0} \sigma_{0}^{+}\right)+[(1+\tau) q+1] \operatorname{Tr}\left(G_{0} \sigma_{0}^{+}\right)=0 .
$$


The change due to initial CS-parameter variation is given by $\Delta F=\operatorname{Tr}\left(G_{0} \Delta \sigma_{0}^{+}\right) \operatorname{Tr}\left(\tilde{G}_{0} \sigma_{0}^{+}\right)+$ $\operatorname{Tr}\left(G_{0} \sigma_{0}^{+}\right) \operatorname{Tr}\left(\tilde{G}_{0} \Delta \sigma_{0}^{+}\right)$. Inserting Eq. (38) and setting $\Delta F=0$ yields

$$
\operatorname{Tr}\left(\left\{[(1+\tau) q+1] G_{0}-(q+1) \tilde{G}_{0}\right\} \Delta \sigma_{0}^{+}\right)=0 .
$$

Inserting the expression for $\tilde{G}_{0}$, Eqs. (29) and (30), this equation reduces to $\operatorname{Tr}\left(G_{0}(q) \Delta \sigma_{0}^{+}\right)=0$ with $G_{0}(q)$ given by Eq. (31). This is the same equation for minimizing $\operatorname{Tr}\left(G_{0}(q) \sigma_{0}^{+}\right)$as long as $\left|G_{0}(q)\right|>0$; thus the solution simply reads, as discussed before,

$$
\sigma_{0}^{\mathrm{opt}}=G_{0}(q) / \sqrt{\left|G_{0}(q)\right|} .
$$

Under this optimal condition, Eq. (38) reduces to

$$
\begin{aligned}
\operatorname{Tr}\left((q+1) G_{0}\left[(1+\tau) q^{2}+2 q\right] G_{0}^{+}(q)\right. \\
\left.+[(1+\tau) q+1] G_{0}\left(q^{2}+2 q\right) G_{0}^{+}(q)\right)=0 .
\end{aligned}
$$

Using Eq. (31), we see that the parameter of this equation depends on a lattice through $\operatorname{Tr}\left(A A^{+}\right)=2|A|, \operatorname{Tr}\left(A B^{+}\right)=$ $-\operatorname{Tr}(A J B J)$, and $\operatorname{Tr}\left(B B^{+}\right)=0$. Thus, after little algebra, the equation for the optimal $q$ finally reduces to a cubic equation $[5,9]$,

$$
(1+\tau) q^{3}+2(2+\tau) q^{2}+[3+(2+\tau) / c] q+2 / c=0,
$$

where $c$ is defined in Eq. (32).

The nature of the roots of the cubic equation depends on its discriminant

$$
D=\frac{4}{c^{3}}(c-1)(1+\tau-c)\left[(2+\tau)^{3}-9\left(1+\tau+\tau^{2}\right) c\right] .
$$

Depending on the sign of $D$, there can be one to three real solutions. However, only one satisfies $\left|G_{0}(q)\right|>0$, i.e., $q c>-1$. See Fig. 5 for the viable solutions of Eq. (42) over the $\tau-c$ parameter space.

Putting the solution $q^{\text {opt }}$ of Eq. (42) into Eqs. (30) and (40) gives the optimal dispersion and C-S parameters at the dipole entrance. The resulting minimum emittance is given by (with $q=q^{\text {opt }}$ )

$$
F^{\min }=\frac{\operatorname{Tr}\left[G_{0}\left(q^{2}+2 q\right) G_{0}^{+}(q)\right] \operatorname{Tr}\left\{G_{0}\left[(1+\tau) q^{2}+2 q\right] G_{0}^{+}(q)\right\}}{\left|G_{0}(q)\right|}=4|A| \frac{[1+(q+3) q c / 2]\{1+[(1+\tau) q+3] q c / 2\}}{1+q c}
$$

where Eq. (26) has been used to compute $\left|G_{0}(q)\right|$.

For conventional uniform dipoles without transverse gradient, $\tau=1 / 2$ and $c \simeq 8 / 9$, there is a unique real solution $q^{\text {opt }} \simeq-0.85$ (versus 0 and -1 for achromatic and TME conditions, respectively). The resulted $F^{\mathrm{min}} \simeq$ $4|A|(0.267)$, and thus the emittance is $3 \sqrt{0.267} \simeq 1.55$ times of the TME value of the betatron emittance as was found in [5].

\section{Sandwich dipole}

Several numerical and analytical studies have been done to investigate the potential reduction of emittance with a nonuniform dipole $[8,9,11]$. A three-step model without central symmetry has been investigated numerically for lattice upgrade of the European Synchrotron Radiation Facility [15]. Here we consider a simplified analytically solvable model that replaces the uniform dipole with a nonuniform dipole made of three adjacent uniform sector dipoles, where the middle dipole of radius $\rho_{0}$ and bending angle $\theta_{0}$ is sandwiched by two identical dipoles of radius $\rho_{1}$ and bending angle $\theta_{1}$. Let $\mu=\rho_{1} / \rho_{0}$ and $\nu=\theta_{1} / \theta_{0}$, then $\theta_{0}=\theta /(1+2 \nu)$ and $\theta_{1}=\nu \theta /(1+2 \nu)$, where $\theta$ is the total bending angle of the sandwiched dipole. To compute $\langle\langle H\rangle\rangle_{\min }$, we use the center of the middle dipole as the reference point for computation. Within the middle dipole, the $\hat{\boldsymbol{\xi}}$ is given by Eq. (19) with $k=1$. Within the end dipoles, it is given by

$$
\hat{\boldsymbol{\xi}}=\hat{\boldsymbol{\xi}}_{m}\left(\theta_{0} / 2\right)+M_{m}^{-1} \hat{\boldsymbol{\xi}}_{e}=\left[\begin{array}{c}
\rho_{0}\left(\cos \frac{\theta_{0}}{2}-1\right)+\rho_{1}(\cos \theta-1) \cos \frac{\theta_{0}}{2}-\rho_{0} \sin \theta \sin \frac{\theta_{0}}{2} \\
\sin \frac{\theta_{0}}{2}+\frac{\rho_{1}}{\rho_{0}}(\cos \theta-1) \sin \frac{\theta_{0}}{2}+\sin \theta \cos \frac{\theta_{0}}{2}
\end{array}\right]
$$

where $\hat{\boldsymbol{\xi}}_{m}$ and $\hat{\boldsymbol{\xi}}_{e}$ are the dispersion generating vectors in the middle and the end dipoles, respectively. $M_{m}$ is the transfer matrix from the center to the end of the middle dipole. As was done for a uniform dipole in Sec. II B, it is straightforward to compute the minimum emittance, but the computation is tedious and the exact results are messy. Thus the detailed computations were carried out with MATHEMATICA [16]. The ratio of theoretical minimum emittances in a sandwich dipole to that in a uniform dipole of the same bending angle is approximately given by, to the leading order in small-angle approximation,

$$
\frac{\langle\langle\mathcal{H}\rangle\rangle_{\mathrm{TME}}^{\text {sandwich }}}{\langle\langle\mathcal{H}\rangle\rangle_{\mathrm{TME}}^{\mathrm{Tniform}}} \simeq \sqrt{\frac{\left(\mu^{2}+6 \nu+12 \nu^{2}+8 \nu^{3}\right) f}{\mu^{2}\left(\mu^{2}+2 \nu\right)(\mu+2 \nu)^{2}(1+2 \nu)^{6}}}
$$




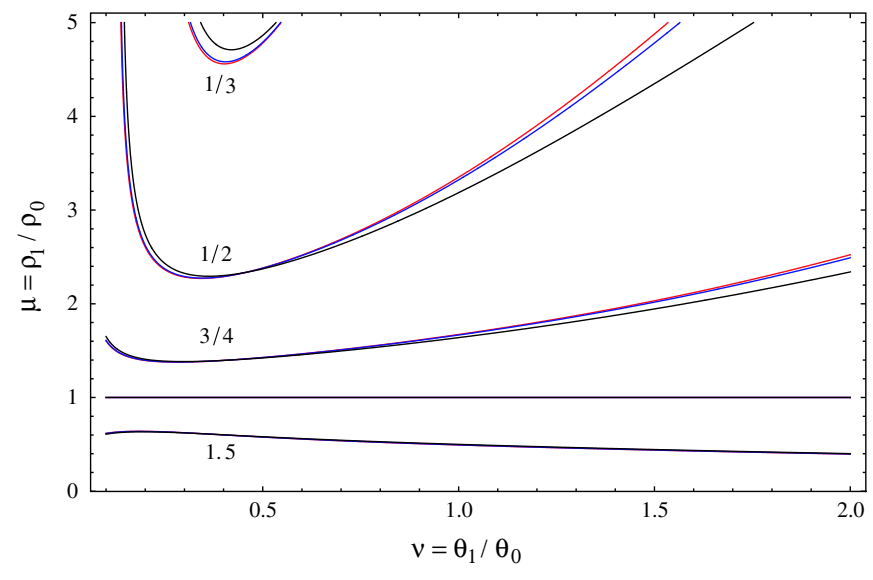

FIG. 2. (Color) Contours of the ratio of the absolute minimum emittances in a sandwich dipole to that in a uniform dipole of the same bending angle. The red curve is the approximation given by Eq. (46). The blue (black) curve is the exact result corresponding to a total bending angle of $\pi / 5(\pi / 2)$.

where the shorthand symbol $f=\mu^{4}\left(1+72 \nu^{5}\right)+$ $20 \mu^{3} \nu^{3}(2+9 \nu)+4 \mu^{2} \nu\left(3+15 \nu+30 \nu^{2}+16 \nu^{5}\right)+$ $120 \mu \nu^{5}+60 \nu^{4}$. To visualize this function, its contour lines are shown in Fig. 2, together with the exact results for bending angles at $\pi / 5$ and $\pi / 2$ which show a negligible dependence on bending angle in general. Indeed, using nonuniform dipoles, it is possible to reduce the emittance below the well-known theoretical minimum emittance. For example, to gain a factor of 2 emittance reduction with sandwich dipoles, one can choose $\mu \simeq 2.3$ and $\nu \simeq 1 / 4$ with an equivalent uniform bending radius $L / \theta=\rho_{0}(1+2 \mu \nu) /(1+2 \nu) \simeq 1.4 \rho_{0}$, which requires the center dipole to be $40 \%$ stronger or the total dipole length $40 \%$ longer. Since beam energy spread is proportional to $\sqrt{\check{\rho}}$, the energy spread change is given by

$$
\frac{\sigma_{\delta}^{\text {sandwich }}}{\sigma_{\delta}^{\text {uniform }}}=\sqrt{\frac{\check{\rho}^{\text {sandwich }}}{\check{\rho}^{\text {uniform }}}}=\sqrt{\frac{(1+2 \mu \nu)\left(\mu^{2}+2 \nu\right)}{(1+2 \nu)(\mu+2 \nu) \mu}},
$$

which is 1.14 for our example, i.e., a $14 \%$ increase of energy spread. With much stronger center dipole, it is seemingly possible to reach lower emittance but there is a limit for a sandwich dipole. By setting $\mu \rightarrow \infty$, Eq. (46) reduces to $\sqrt{\left(1+72 \nu^{5}\right)} /(1+2 \nu)^{3}$ which has a minimum 0.22 at $\nu \simeq 0.45$. In comparison, a numerically optimized dipole with 32 equal-length slices reached a factor of $1 / 7 \simeq$ 0.14 reduction in TME emittance [8].

To better characterize a sandwich dipole, we computed the two key quantities for emittance minimization, i.e., $|A|$ and $c=\operatorname{Tr}\left(A B^{+}\right) /|A|$. The expression is quite messy and given as an appendix. Figure 3 shows the contours of $2 \sqrt{|A|} / \frac{\theta^{3}}{4 \sqrt{15}}$ in red and $c$ in blue, which gives a clear picture of what a sandwich dipole is capable of. From this and Eq. (44) or Fig. 6, we get the effective emittance reduction factor 0.71 for the above example with $\mu=2.3$ and $\nu=$

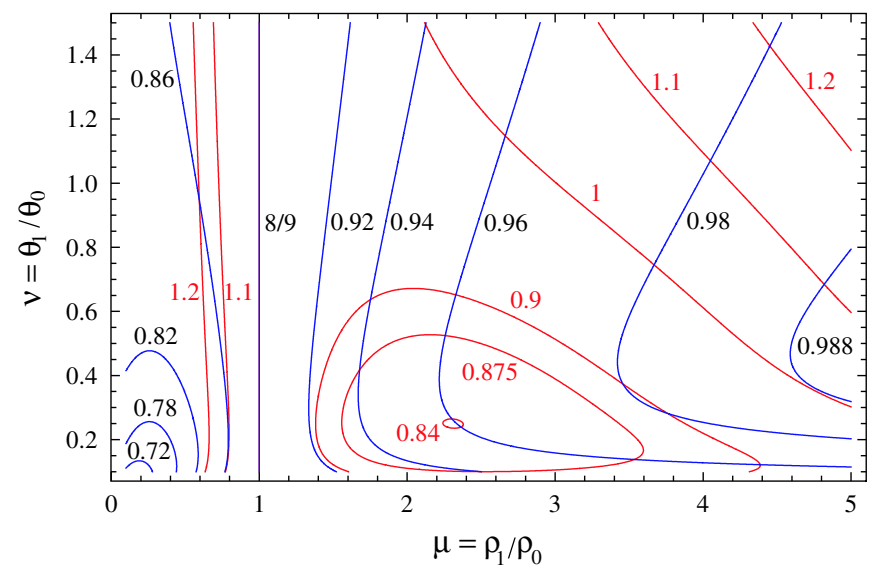

FIG. 3. (Color) Contours of $c=\operatorname{Tr}\left(A B^{+}\right) /|A|$ in blue with black labels and $2 \sqrt{|A|} / \frac{\theta^{3}}{4 \sqrt{15}}$ in red for sandwich dipoles.

$1 / 4$, versus a factor of 0.5 for the TME betatron emittance reduction. In comparison, a numerically optimized threestep dipole without central symmetry can reduce the effective emittance by more than a factor of 2 [15] because it can better fit the asymmetric optimal lattice functions for the minimum effective emittance.

\section{Magnetic field}

To see the impact of varying bending radius on the magnetic field, we write down the extra terms due to radius variation in the general field expansion around the reference orbit in order to satisfy Maxwell's equation up to the third (octupole) order [17]:

$$
\begin{aligned}
& B_{x}=\frac{1}{6}\left(2 \kappa b_{0}^{\prime \prime}+\kappa^{\prime} b_{0}^{\prime}\right) y^{3} \\
& B_{y}=b_{0}-\frac{1}{2} b_{0}^{\prime \prime} y^{2}+\frac{1}{2}\left(2 \kappa b_{0}^{\prime \prime}+\kappa^{\prime} b_{0}^{\prime}\right) x y^{2} \\
& B_{s}=b_{0}^{\prime} y-\kappa b_{0}^{\prime} x y+\kappa^{2} b_{0}^{\prime} x^{2} y-\frac{1}{6} b_{0}^{\prime \prime \prime} y^{3},
\end{aligned}
$$

where we have included only one independent component, i.e., the dipole component $b_{0}(s)$. The orbit curvature $\kappa(s)=1 / \rho(s)$ is tied to the bending field by $\kappa(s)=$ $e b_{0}(s) / p_{s}$, where $e$ and $p_{s}$ are the electron charge and longitudinal momentum. The primes indicate differentiation with respect to longitudinal position $s$. We see that dipole radius variation will introduce a nonlinear magnetic field, whose effects are beyond the scope of this paper. Nonetheless, if we simply integrate all the coefficients across the dipole, all the terms become zero except for the integrated dipole and an integrated octupole-like term proportional to $\int\left(2 \kappa b_{0}^{\prime \prime}+\kappa^{\prime} b_{0}^{\prime}\right) d s=-\left(p_{s} / e\right) \int(1 / \rho)^{\prime 2} d s$, which has to be sufficiently small.

\section{EFFECTS OF DAMPING PARTITION AND NONUNIFORM DIPOLE ON THE EFFECTIVE EMITTANCE}

As discussed in Sec. III B, minimization of the effective emittance is more constrained due to mixing of betatron 
and dispersive motions. Because of the importance of the effective emittance for light sources, it is worthwhile examining how a transverse gradient and bending radius variation can affect it.

\section{A. Effects of transverse gradient in uniform dipole}

With typical bending angles and reasonable transverse gradients, a combined-function uniform dipole has little effect on the minimization of the betatron emittance through $\langle\mathcal{H}\rangle$ as shown in Eq. (23). A gradient can change the horizontal and longitudinal damping partition $J_{x}$ and $J_{E}$ more effectively, and the minimum betatron emittance is simply inversely proportional to $J_{x}$. This is not true anymore for the effective emittance because the minimization becomes sensitive to the damping partition via Eq. (42).

For uniform dipoles, Eq. (27) shows that $c \simeq 8 / 9$ except for unusually large bending angle and transverse gradient. To see the effect of transverse gradient on the minimum effective emittance $\epsilon_{\min }^{\text {eff }}$, we use $c=8 / 9$ in Eq. (42) and compute the optimal $q$ and $\epsilon_{\text {min }}^{\text {eff }} / C_{q} \gamma^{2} 2 \sqrt{|A|}=$ $\sqrt{F^{\min } / 4|A|} / J_{x}$ for a given partition $J_{x} / J_{E}$. The results are plotted in Fig. 4. It shows that the minimum effective emittance (solid curve) decreases with increasing $J_{x}$, but much slower than the $1 / J_{x}$ dependence (dashed curve). In other words, gradient dipoles are less effective at reducing the effective emittance. (Besides emittance reduction, gradient dipoles may be useful for other purposes in optics design.)

\section{B. Effects of nonuniform dipoles}

For nonuniform dipoles with bending radius variation, its $c$ parameter can change significantly from $8 / 9$. It is of interest to understand its effect on the minimum effective emittance, in addition to the effects of damping partition

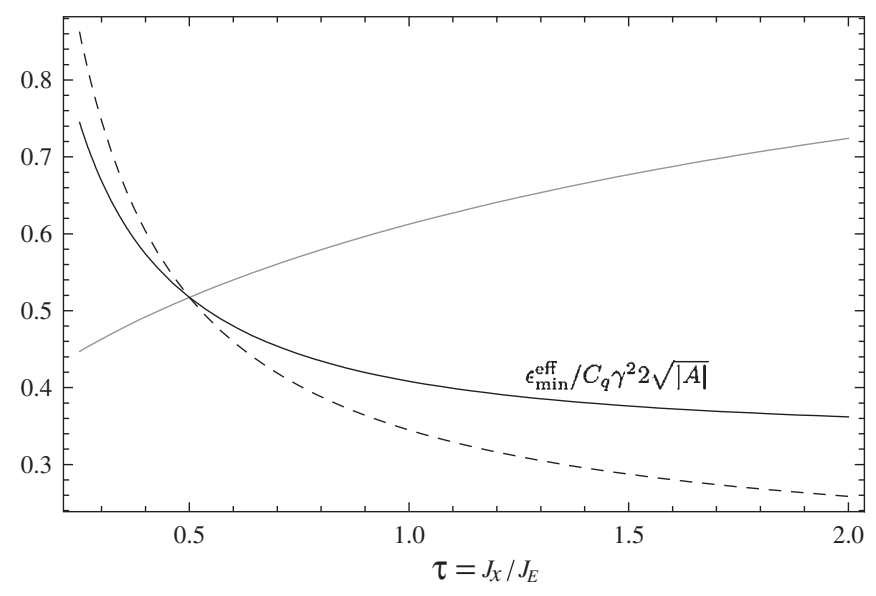

FIG. 4. Change of the minimum effective emittance with damping partition variation for uniform dipoles. The solid, gray, and dashed curves show, respectively, $\sqrt{F^{\mathrm{min}} / 4|A|} / J_{x}$, $\sqrt{F^{\min } / 4|A|}$, and $\left.\sqrt{F^{\min } / 4|A|}\right|_{J_{x}=1} / J_{x}$.

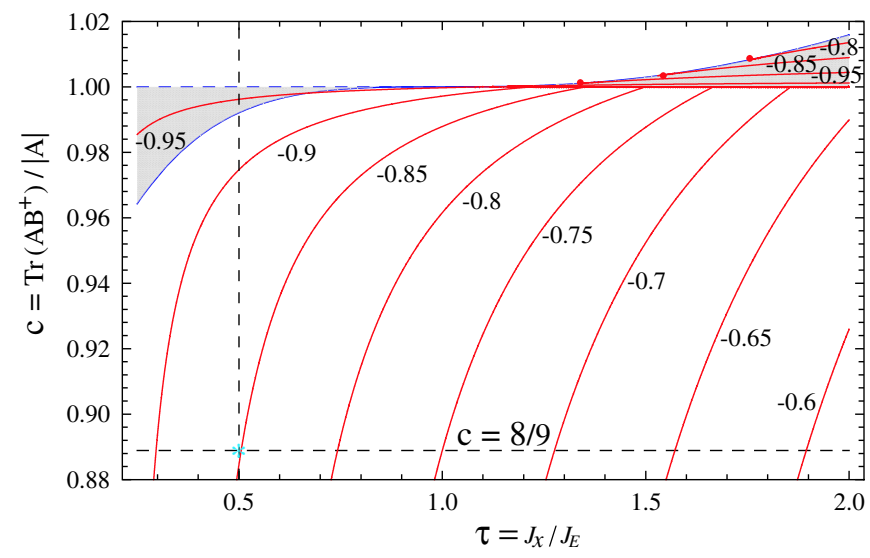

FIG. 5. (Color) Contours of optimal $q$ (red curves) for minimum effective emittance over the $\tau-c$ parameter space. The gray (white) area shows the region with positive (negative) discriminant for the cubic equation. Only one of the three roots in the gray area is a suitable solution.

variation discussed above. To explore the $\tau$-c parameter space for effective emittance optimization, for each $\tau$ and $c$, we determine the optimal $q$ by solving the cubic equation (42) and compute $\epsilon_{\min }^{\text {eff }} / C_{q} \gamma^{2} 2 \sqrt{|A|}=\sqrt{F^{\mathrm{min}} / 4|A|} / J_{x}$ with Eq. (44), whose contours over the $\tau$-c parameter space are plotted in Figs. 5 and 6. The nominal working point in a typical storage ring is at the intersect of the two black dashed lines. Increasing $\tau$ or $c$ can both reduce the effective emittance but the gain is rather limited. In addition to the factor shown in Fig. 6, both betatron and effective emittances can benefit from reduction of $|A|$ with a nonuniform dipole. For a sandwich dipole discussed above, the maximum reduction in $\sqrt{|A|}$ is a factor of 0.84 .

As the $c$ parameter approaches $1, q^{\text {opt }} \rightarrow-1$ which is the same value required for a TME lattice. However, there is a major difference. In the TME case, the betatron emit-

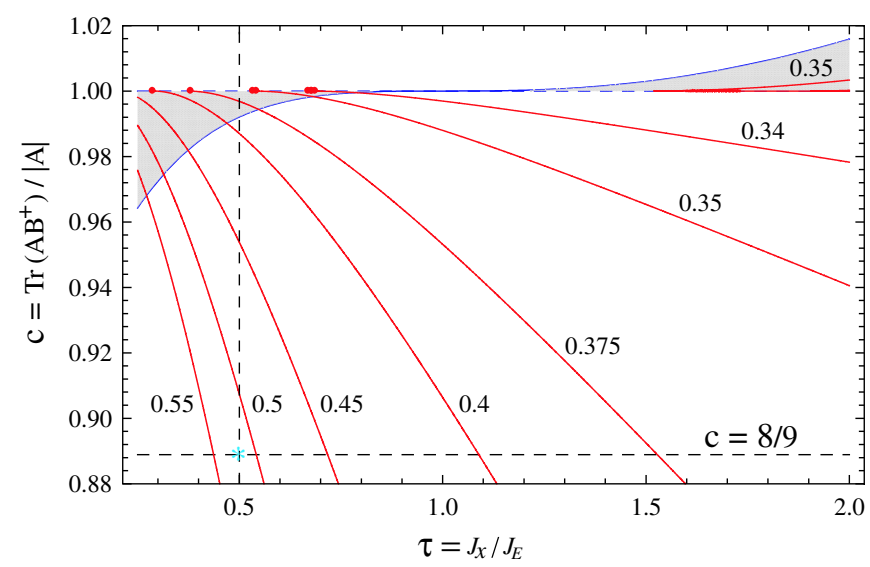

FIG. 6. (Color) Contours of minimum effective emittance $\epsilon_{\min }^{\mathrm{eff}} / C_{q} \gamma^{2} 2 \sqrt{|A|}$ (red curves) over the $\tau-c$ parameter space. The horizontal and vertical black dashed lines give $c=8 / 9$ and $\tau=1 / 2$, respectively. 
tance is proportional to $\sqrt{|A-B|}=\sqrt{|A|(1-c)}$, which can in principle approach zero as $c \rightarrow 1$. But the effective emittance can never approach zero due to the contribution from beam energy spread.

\section{CONCLUSION}

An elegant and complete theory of minimum emittance in storage rings with arbitrary dipoles is presented, which offers a new viewpoint to this matured subject. Using vector and matrix forms, concise and general expressions for minimum emittance as well as the optimal lattice parameters are derived without any assumptions on the transport matrix inside a dipole. Minimization of both natural and effective emittances are treated for dipoles with transverse gradient and/or longitudinal field variation. Simple formulas are derived for evaluating the impact of nonoptimal-lattice parameters, which shows the importance of keeping the same enlargement factor for both beta and alpha functions at the dipole entrance. New parameters solely determined by a magnet are introduced to uniquely characterize the performance of a dipole for emittance minimization. Effects of dipole parameters as well as damping partition numbers on the effective emittance are analyzed over parameter spaces of general interest. Because of its conciseness and simplicity, the presented theory should have pedagogical value as well.

\section{ACKNOWLEDGMENTS}

I thank my colleagues T. C. Lee and L. Emery for helpful discussions and comments. This work was supported by U.S. Department of Energy, Office of Science, Office of Basic Energy Sciences, under Contract No. DE-AC0206CH11357.

\section{APPENDIX A: $|A|, c$ OF SANDWICH DIPOLES}

For completeness, we include the formulas used to generate Fig. 3 in the text. These are computed with MATHEMATICA and contains only the leading-order terms in small-angle expansion:

$$
|A|=\left|\left\langle\left\langle\hat{\boldsymbol{\xi}} \hat{\boldsymbol{\xi}}^{T}\right\rangle\right\rangle\right|=\frac{\theta^{6}}{2880 \mu^{2}(2 \nu+1)^{6}(\mu+2 \nu)^{2}} f_{1},
$$

where $f_{1}=768 \mu^{2} \nu^{8}+768 \mu^{4} \nu^{7}+1024 \mu^{2} \nu^{7}+1280 \mu \nu^{7}+$ $1008 \mu^{4} \nu^{6}+1680 \mu^{3} \nu^{6}+448 \mu^{2} \nu^{6}+1680 \mu \nu^{6}+560 \nu^{6}+$ $496 \mu^{4} \nu^{5}+2480 \mu^{3} \nu^{5}+960 \mu^{2} \nu^{5}+720 \mu \nu^{5}+720 \nu^{5}+$ $60 \mu^{6} \nu^{4}+1400 \mu^{3} \nu^{4}+1600 \mu^{2} \nu^{4}+300 \nu^{4}+120 \mu^{5} \nu^{3}+$ $160 \mu^{3} \nu^{3}+1064 \mu^{2} \nu^{3}+20 \mu^{5} \nu^{2}+64 \mu^{4} \nu^{2}+252 \mu^{2} \nu^{2}+$ $24 \mu^{4} \nu+24 \mu^{2} \nu+3 \mu^{4}$.

$$
c=\frac{\operatorname{Tr}\left(A B^{+}\right)}{|A|}=\frac{4 f_{2}}{3\left(\mu^{2}+2 \nu\right) f_{1}},
$$

where $f_{2}=1024 \mu^{2} \nu^{9}+1584 \mu^{4} \nu^{8}+1344 \mu^{2} \nu^{8}+$ $1680 \mu \nu^{8}+576 \mu^{6} \nu^{7}+2064 \mu^{4} \nu^{7}+3120 \mu^{3} \nu^{7}+$ $576 \mu^{2} \nu^{7}+2160 \mu \nu^{7}+720 \nu^{7}+756 \mu^{6} \nu^{6}+1260 \mu^{5} \nu^{6}+$ $956 \mu^{4} \nu^{6}+4360 \mu^{3} \nu^{6}+1620 \mu^{2} \nu^{6}+900 \mu \nu^{6}+900 \nu^{6}+$ $444 \mu^{6} \nu^{5}+1860 \mu^{5} \nu^{5}+720 \mu^{4} \nu^{5}+2220 \mu^{3} \nu^{5}+$ $2460 \mu^{2} \nu^{5}+360 \nu^{5}+45 \mu^{8} \nu^{4}+1185 \mu^{5} \nu^{4}+1200 \mu^{4} \nu^{4}+$ $180 \mu^{3} \nu^{4}+1422 \mu^{2} \nu^{4}+90 \mu^{7} \nu^{3}+140 \mu^{5} \nu^{3}+$ $862 \mu^{4} \nu^{3}+252 \mu^{2} \nu^{3}+15 \mu^{7} \nu^{2}+48 \mu^{6} \nu^{2}+$ $207 \mu^{4} \nu^{2}+18 \mu^{2} \nu^{2}+18 \mu^{6} \nu+18 \mu^{4} \nu+2 \mu^{6}$.

These reduce to $|A|=\theta^{6} / 960$ and $c=8 / 9$ when $\mu=$ 1 , the result for a uniform dipole.

[1] M. Sommer, Internal Report No. DCI/NI/20/81, 1981. See references in [4] for more internal reports in the early years.

[2] L. Teng, FNAL Report No. FNAL/TM-1269, 1984; ANL Report No. LS-17, 1985.

[3] D. Trbojevic and E. Courant, in Proceedings of the 4th European Particle Accelerator Conference, London, 1994 (EPS-IGA and CERN, Geneva, 1994), p. 1000.

[4] S. Y. Lee, Phys. Rev. E 54, 1940 (1996).

[5] H. Tanaka and A. Ando, Nucl. Instrum. Methods Phys. Res., Sect. A 369, 312 (1996).

[6] Y. Shoji and A. Ando, Nucl. Instrum. Methods Phys. Res., Sect. A 425, 377 (1999).

[7] T. Y. Lee and J. Choi, Nucl. Instrum. Methods Phys. Res., Sect. A 534, 371 (2004).

[8] J. Guo and T. Raubenheimer, in Proceedings of the 8th European Particle Accelerator Conference, Paris, 2002 (EPS-IGA and CERN, Geneva, 2002), p. 1136.

[9] Y. Papaphilippou and P. Elleaume, in Proceedings of the 21st Particle Accelerator Conference, Knoxville, 2005 (IEEE, Piscataway, NJ, 2005), p. 2086.

[10] A. Streun, PSI Internal Report No. SLS-TME-TA-20060297, 2007.

[11] R. Nagaoka and A. F. Wrulich, Nucl. Instrum. Methods Phys. Res., Sect. A 575, 292 (2007).

[12] See, for example, M. Sands, SLAC Report No. SLAC-121, 1970.

[13] R. H. Helm, M. J. Lee, P.L. Morton, and M. Sands, in Proceedings of the 1973 Particle Accelerator Conference, San Francisco, CA, 1973 [IEEE Trans. Nucl. Sci. 20, 900 (1973)].

[14] Taking trace on both sides of the Schur decomposition gives $\langle\mathcal{H}\rangle$ on the left-hand side and $\lambda_{1}+\lambda_{2}$ on the righthand side since $V$ and $V^{-1}$ cancel each other. Similarly, taking the determinant on both sides gives $\left|G_{0}\right|$ on the lefthand side since $\left|\mathcal{A}_{0}\right|=1$ and $\lambda_{1} \lambda_{2}$ on the right-hand side.

[15] Y. Papaphilippou, A. Ropert, P. Elleaume, and L. Farvacque, in Ref. [9], p. 2047.

[16] http://www.wolfram.com.

[17] C.-x. Wang and L. Teng, in Proceedings of the 2001 Particle Accelerator Conference, Chicago, IL (IEEE, Piscataway, NJ, 2001), p. 456. 